\title{
Observations on the Stability Properties of
}

\section{Cooperative Systems}

\author{
Oliver Mason and Mark Verwoerd
}

\begin{abstract}
We extend two fundamental properties of positive linear time-invariant (LTI) systems to homogeneous cooperative systems. Specifically, we demonstrate that such systems are $D$-stable, meaning that global asymptotic stability is preserved under diagonal scaling. We also show that a delayed homogeneous cooperative system is globally asymptotically stable (GAS) for any non-negative delay if and only if the system is GAS for zero delay.
\end{abstract}

\section{INTRODUCTION}

Positive dynamical systems, in which the state variables are constrained to remain non-negative, arise in numerous application areas. In fact, any situation in which the variables of interest only take non-negative values gives rise to a positive dynamical system. Examples of this type can be found in areas such as Ecology (population sizes), Biology (gene/protein concentrations), Economics (commodity prices) and Chemical Engineering (chemical concentrations).

Essentially a positive dynamical system is one for which non-negative initial conditions always give rise to non-negative trajectories. Given their practical importance, it is not surprising that a considerable deal of attention has been paid to the study of positive systems and to the elucidation of their basic properties. At the time of writing, there is a well developed theory of positive linear time-invariant (LTI) systems, with roots in the Perron-Frobenius theory of non-negative matrices [6], [3]. Particular attention has been paid to questions relating to positive reachability and observability, and the existence of positive realizations of transfer functions. As with any system class, issues pertaining to stability and the existence and location of equilibria are of fundamental importance. The work described here focusses on this aspect of the theory of positive systems. 
In the linear time-invariant case, it is well known that if the origin is a globally asymptotically stable (GAS) equilibrium of the positive LTI system $\dot{x}(t)=A x(t)$, then it is also a GAS equilibrium of $\dot{x}(t)=D A x(t)$ for all diagonal matrices $D$ with positive elements on the diagonal. This property is commonly referred to as $D$-stability. More recently, it was shown in [8] that for a positive linear delayed system $\dot{x}(t)=A x(t)+B x(t-\tau)$, the origin is a GAS equilibrium for any $\tau \geq 0$ if and only if it is a GAS equilibrium of the system with zero delay $\dot{x}(t)=(A+B) x(t)$. The two main results of the current paper will show that these two properties of positive LTI systems naturally extend to an important class of nonlinear positive systems.

Specifically, we shall show that these results extend to the class of homogeneous cooperative systems. Cooperative systems are a particularly important subclass of nonlinear positive systems and have been studied extensively, particularly in relation to biological applications of dynamical systems [16], [12]. A key property of such systems, which also holds for positive LTI systems, is that they are monotone [16], [5]. Essentially this means that if we consider two initial vectors $x_{0}$ and $y_{0}$ where $x_{0}$ is less than $y_{0}$ componentwise, then the trajectory starting from $x_{0}$ remains less than that starting from $y_{0}$ (componentwise) for all subsequent times. Recently, motivated by applications in Cell Biology, the basic theory of monotone dynamical systems has been extended to consider interconnections of such systems and a control theory of monotone systems has been developed in the papers [2], [1]. While the result for delayed positive linear systems in [8] was derived using Lyapunov-Krasovskii techniques, the methods adopted here are based on the fundamental monotonicity properties of cooperative systems. As such, in addition to extending the result in [8], we provide an alternative view on it.

Other recent work on nonlinear positive systems has been presented in [9]. In this paper, the stability and dissipativity properties of nonlinear, not necessarily cooperative, positive systems have been investigated. Further, motivated by applications in anaesthesiology, adaptive control methods for nonlinear positive systems have been proposed and analysed in [10].

The layout of the paper is as follows. In the next section, we introduce notation and some mathematical background. In Section III we prove that homogeneous cooperative systems possess the $D$-stability property. In Section IV we consider systems subject to delay and present a nonlinear extension of the result described in the previous paragraph. Finally, in Section V we give some concluding remarks. 


\section{NOTATION AND BACKGROUND}

Throughout the paper, $\mathbb{R}$ and $\mathbb{R}^{n}$ denote the field of real numbers and the vector space of all $n$-tuples of real numbers respectively. $\mathbb{R}^{n \times n}$ denotes the space of $n \times n$ matrices with real entries. For $x \in \mathbb{R}^{n}$ and $i=1, \ldots, n, x_{i}$ denotes the $i^{t h}$ coordinate of $x$. Similarly, for $A \in \mathbb{R}^{n \times n}, a_{i j}$ denotes the $i, j$ entry of $A$.

In the interest of brevity, we shall slightly abuse notation and refer to a system as being GAS when the origin is a GAS equilibrium of the system. Also, as we are dealing with positive systems throughout, when we refer to a system as GAS, it is with respect to initial conditions in $\mathbb{R}_{+}^{n}$, where $\mathbb{R}_{+}^{n}$ is the set of all vectors in $\mathbb{R}^{n}$ with non-negative entries,

$$
\mathbb{R}_{+}^{n}:=\left\{x \in \mathbb{R}^{n}: x_{i} \geq 0,1 \leq i \leq n\right\}
$$

For vectors $x, y \in \mathbb{R}^{n}$, we write: $x \geq y$ if $x_{i} \geq y_{i}$ for $1 \leq i \leq n$; $>>y$ if $x \geq y$ and $x \neq y$; $x \gg y$ if $x_{i}>y_{i}, 1 \leq i \leq n$.

A matrix $A \in \mathbb{R}^{n \times n}$ is said to be non-negative if $a_{i j} \geq 0$ for $1 \leq i, j \leq n$. Similarly, a vector field $g: \mathbb{R}^{n} \rightarrow \mathbb{R}^{n}$ is non-negative if $g(x) \geq 0$ for all $x \in \mathbb{R}_{+}^{n}$.

For a real interval $[a, b], C\left([a, b], \mathbb{R}_{+}^{n}\right)$ denotes the space of all real-valued continuous functions on $[a, b]$ taking values in $\mathbb{R}_{+}^{n}$. For functions $f, g \in C\left([a, b], \mathbb{R}_{+}^{n}\right)$ we write $f \geq g$ if $f(s) \geq$ $g(s), \forall s \in[a, b]$.

Throughout the paper, for a vector $x \in \mathbb{R}^{n},\|x\|$ denotes the usual Euclidean norm of $x$. For a matrix $A \in \mathbb{R}^{n \times n},\|A\|$ denotes the matrix norm induced by the Euclidean norm. Finally, for $d=\left(d_{1}, \ldots, d_{n}\right)^{T}$ in $\mathbb{R}^{n}, \operatorname{diag}\left(d_{1}, \ldots, d_{n}\right)$ denotes the diagonal matrix in $\mathbb{R}^{n \times n}$ in which the $i^{t h}$ entry on its main diagonal is $d_{i}$.

\section{A. Positive linear systems}

In this subsection, we recall some basic facts concerning positive LTI systems.

Definition 2.1: The LTI system

$$
\dot{x}(t)=A x(t)
$$

is positive if $x_{0} \geq 0 \Rightarrow x\left(t, x_{0}\right) \geq 0$ for all $t \geq 0$, where $x\left(\cdot, x_{0}\right)$ denotes the unique solution of (1) satisfying $x\left(0, x_{0}\right)=x_{0}$. 
It is well known and easily verified that an LTI system is positive if and only if its system matrix $A$ satisfies $a_{i j} \geq 0$ for $i \neq j$. Matrices of this form are said to be Metzler. Positive LTI systems are automatically monotone due to linearity. Formally, if $x_{0} \leq y_{0}$, then $x\left(t, x_{0}\right) \leq x\left(t, y_{0}\right)$ for all $t \geq 0$.

For convenience, we collect some standard facts concerning the stability of positive LTI systems in the following result.

Theorem 2.1: Consider the positive LTI system (1). The following statements are equivalent:

(i) The system (1) is globally asymptotically stable;

(ii) There is some vector $v \gg 0$ such that $A v \ll 0$;

(iii) $\dot{x}(t)=D A x(t)$ is globally asymptotically stable for all diagonal matrices $D=\operatorname{diag}\left(d_{1}, \ldots, d_{n}\right)$ with $d_{i}>0$ for $1 \leq i \leq n$.

The property described by point (iii) above is usually referred to as D-stability.

In the recent papers [8], [14] the stability properties of delayed positive linear systems were studied. Formally, consider the system

$$
\dot{x}(t)=A x(t)+B x(t-\tau), \quad \tau>0 .
$$

Recall that for delay systems of this form [11], for any initial conditions given by a function $\phi \in C\left([-\tau, 0], \mathbb{R}_{+}^{n}\right)$ there exists a unique solution $x(t, \phi)$ defined for $t \in[-\tau, \infty)$, satisfying (2) and $x(s, \phi)=\phi(s)$ for $s \in[-\tau, 0]$. Typically, the history segment $x_{t}:[-\tau, 0] \rightarrow \mathbb{R}^{n}$ given by $x_{t}(s)=x(t-s)$ for $-\tau \leq s \leq 0$ is referred to as the state of the system at time $t$.

As with LTI systems, the system (2) is positive if $\phi \geq 0$ implies $x(t, \phi) \geq 0$ for all $t \geq 0$. It has been shown in [8] that (2) is positive if and only if $A$ is Metzler and $B$ is non-negative. As in the undelayed case, it follows from linearity that the flows generated by a positive delayed linear system are monotone meaning that $\phi \leq \psi$ implies $x(t, \phi) \leq x(t, \psi)$ for all $t \geq 0$.

The following result from [8] shows that (2) is globally asymptotically stable for any $\tau \geq 0$ if and only if the undelayed system with $\tau=0$ is globally asymptotically stable.

Theorem 2.2: The time-delayed positive linear system (2) is globally asymptotically stable for any $\tau \geq 0$ if and only if

$$
\dot{x}(t)=(A+B) x(t)
$$

is globally asymptotically stable. 
Remark: Theorem 2.2 is a slight rewording of Theorem 3.1 of [8]. A corresponding result for exponential stability of delay systems was subsequently established in Theorem 4.1 of [14]. The proof in [8] relies on the theory of Lyapunov-Krasovskii functionals, while the proof for exponential stability in [14] is based on analysing the characteristic function of the delayed system. The primary contribution of the present paper is to extend both Theorem 2.2 and Theorem 2.1 to a class of nonlinear positive systems using different methods which rely directly on a key property of the system class; namely the monotonicity of solutions with respect to initial conditions. As such, we also provide an alternative perspective on the results in [8], [14].

\section{B. Homogeneous cooperative systems}

In the study of nonlinear positive systems, the class of co-operative systems is of particular importance for numerous applications in Economics, Biology and Ecology and has attracted a considerable deal of attention in the past [16]. The definition of a cooperative vector field is as follows.

Definition 2.2: A continuous vector field $f: \mathbb{R}^{n} \rightarrow \mathbb{R}^{n}$, which is $C^{1}$ on $\mathbb{R}^{n} \backslash\{0\}$ is said to be cooperative if the jacobian $\frac{\partial f}{\partial x}(a)$ is a Metzler matrix for all $a \in \mathbb{R}_{+}^{n} \backslash\{0\}$.

The results presented later in the paper are concerned with cooperative systems whose vector fields are homogeneous in the sense of the following definition.

Definition 2.3: $f: \mathbb{R}^{n} \rightarrow \mathbb{R}^{n}$ is said to be homogeneous if for all $x \in \mathbb{R}^{n}$ and all real $\lambda>0$, $f(\lambda x)=\lambda f(x)$.

Let the vector field $f: \mathbb{R}^{n} \rightarrow \mathbb{R}^{n}$ be continuous on $\mathbb{R}^{n}$ and $C^{1}$ on $\mathbb{R}^{n} \backslash\{0\}$. If $f$ is homogeneous then it follows easily that for any $a \in \mathbb{R}^{n} \backslash\{0\}$ and any $\lambda>0$,

$$
\frac{\partial f}{\partial x}(\lambda a)=\frac{\partial f}{\partial x}(a)
$$

This immediately allows us to conclude the following result, whose proof we include in the interest of completeness.

Lemma 2.1: Let $f: \mathbb{R}^{n} \rightarrow \mathbb{R}^{n}$ be continuous on $\mathbb{R}^{n}, C^{1}$ on $\mathbb{R}^{n} \backslash\{0\}$ and homogeneous. Then there exists $K>0$ such that $\|f(x)-f(y)\| \leq K\|x-y\|$ for all $x, y \in \mathbb{R}^{n}$.

Proof: As $f$ is $C^{1}$, it follows that there is some $K_{1}>0$ such that

$$
\left\|\frac{\partial f}{\partial x}(a)\right\| \leq K_{1}
$$


for all $a$ with $\|a\|=1$. But (4) then implies that this inequality must hold for all $a \neq 0$. Now pick any $x \neq 0$ in $\mathbb{R}^{n}$. It follows from the mean value theorem that for any $y \in \mathbb{R}^{n} \backslash\{t x: t \leq 0\}$,

$$
\|f(x)-f(y)\| \leq K_{1}\|x-y\| .
$$

It now follows from the continuity of $f$ that this inequality must hold for all $y \in \mathbb{R}^{n}$. As $x$ was an arbitrary non-zero vector, it follows that (5) holds for all $x \in \mathbb{R}^{n} \backslash\{0\}, y \in \mathbb{R}^{n}$. It now follows again by continuity that the inequality must hold for all $x, y \in \mathbb{R}^{n}$.

If $f: \mathbb{R}^{n} \rightarrow \mathbb{R}^{n}$ is homogeneous, the previous lemma immediately implies the existence and uniqueness of solutions to the system $\dot{x}(t)=f(x(t))$ for any initial conditions in $\mathbb{R}^{n}$.

We next collect some well-established facts concerning dynamical systems defined by cooperative, homogeneous vector fields. Before stating the result, note that Lemma 5.1 of [5] established the existence of solutions to systems with cooperative, homogeneous right hand sides for all $t \geq 0$.

Theorem 2.3: Let $f: \mathbb{R}^{n} \rightarrow \mathbb{R}^{n}$ be a vector field that is continuous on $\mathbb{R}^{n}$ and $C^{1}$ on $\mathbb{R}^{n} \backslash\{0\}$ and suppose that $f$ is homogeneous and cooperative. Consider the associated dynamical system

$$
\dot{x}(t)=f(x(t))
$$

and for $x_{0} \in \mathbb{R}_{+}^{n}$, let $x\left(\cdot, x_{0}\right)$ denote the solution of (6) satisfying $x\left(0, x_{0}\right)=x_{0}$. Then:

(i) For any real number $\lambda>0$ and any $x_{0} \in \mathbb{R}_{+}^{n}, x\left(t, \lambda x_{0}\right)=\lambda x\left(t, x_{0}\right)$ for any $t \geq 0$;

(ii) For any $x_{0}, x_{1} \in \mathbb{R}_{+}^{n}$, if $x_{0} \leq x_{1}$, it follows that $x\left(t, x_{0}\right) \leq x\left(t, x_{1}\right)$ for any $t \geq 0$.

Proof: Under the hypotheses of the theorem, for any $x_{0} \in \mathbb{R}_{+}^{n}$, there exists a unique solution of (6) through $x_{0}$. This together with the homogeneity of $f$ implies (i). The statement in (ii) was proven in Proposition 4.3 of [5].

Finally for this section, we state a result for delayed systems that corresponds to point (ii) of Theorem 2.3 above. For details consult [16]. First of all, we recall the definition of an order preserving vector field.

Definition 2.4: $g: \mathbb{R}^{n} \rightarrow \mathbb{R}^{n}$ is order-preserving on $\mathbb{R}_{+}^{n}$ if $g(x) \geq g(y)$ for any $x, y \in \mathbb{R}_{+}^{n}$ such that $x \geq y$.

As we shall only be interested in vector fields that are order-preserving on $\mathbb{R}_{+}^{n}$, we shall usually refer to such vector fields as simply order-preserving. 
Theorem 2.4: Let $f, g: \mathbb{R}^{n} \rightarrow \mathbb{R}^{n}$ be continuous on $\mathbb{R}^{n}$ and $C^{1}$ on $\mathbb{R}^{n} \backslash\{0\}$. Further assume that $f$ is cooperative and homogeneous while $g$ is order-preserving and homogeneous. Let $x(\cdot, \phi)$ denote the solution of the delayed system

$$
\dot{x}(t)=f(x(t))+g(x(t-\tau))
$$

corresponding to the initial condition $\phi \in C\left([-\tau, 0], \mathbb{R}_{+}^{n}\right)$. Then for any $\phi, \psi \in C\left([-\tau, 0], \mathbb{R}_{+}^{n}\right)$ with $\phi \leq \psi$, we have that $x(t, \phi) \leq x(t, \psi)$ for all $t \geq 0$ for which both solutions are defined.

Comment: As in the case of undelayed systems, the existence and uniqueness of solutions to (7) is implied by Lemma 2.1 (see Chapter 2 of [11]). With regard to continuation of solutions for the delayed case, we can conclude from Theorem 2.3.2 of [11] that if each trajectory of (7) remains in a compact set in each finite time interval, then the solutions of (7) can be continued to $[0, \infty)$. We shall make use of this fact in the proof of Theorem 4.1 below.

\section{A nonlinear Perron Frobenius Theorem}

Many of the stability properties of positive linear systems are natural consequences of the PerronFrobenius theorem for non-negative matrices. Numerous authors have considered the problem of extending the Perron-Frobenius Theorem to nonlinear positive systems. The appendix in [13] is an excellent early reference on this topic (in finite dimensions), while further and more general results were subsequently reported in [15], [7], [5]. We shall only require a particular case of a recent result presented in [5] for irreducible, homogeneous cooperative systems.

Definition 2.5: $f: \mathbb{R}^{n} \rightarrow \mathbb{R}^{n}$ is said to be irreducible on $\mathbb{R}_{+}^{n}$ if:

(i) $\frac{\partial f}{\partial x}(a)$ is an irreducible matrix for all $a$ in the interior of $\mathbb{R}_{+}^{n}$;

(ii) for non-zero $a$ in the boundary of $\mathbb{R}_{+}^{n}$, either $\frac{\partial f}{\partial x}(a)$ is irreducible or $a_{i}=0$ implies $f_{i}(a)>0$. The paper [5] contains a variety of interesting technical results concerning the asymptotic properties of homogeneous cooperative systems but, for our purposes, the facts collected in the following theorem will prove sufficient.

Theorem 2.5: Let $f: \mathbb{R}^{n} \rightarrow \mathbb{R}^{n}$ be continuous, and $C^{1}$ on $\mathbb{R}^{n} \backslash\{0\}$. Further, assume that $f$ is cooperative, homogeneous and irreducible. Then there exists a unique vector $v \gg 0$ and a real number $\gamma_{v}$ such that $f(v)=\gamma_{v} v$. Moreover the system $\dot{x}(t)=f(x(t))$ is globally asymptotically stable if and only if $\gamma_{v}<0$. 


\section{STABILITY AND D-STABILITY FOR HOMOGENEOUS COOPERATIVE SYSTEMS}

In this section, we shall extend Theorem 2.1 to homogeneous cooperative systems. Firstly, we shall demonstrate the equivalence of (i) and (ii) for this system class. Throughout this section, unless stated otherwise, all vector fields are continuous on the whole of $\mathbb{R}^{n}$ and $C^{1}$ on $\mathbb{R}^{n} \backslash\{0\}$. In proving the main result of this section, we shall need the following technical lemma.

Lemma 3.1: Let $f: \mathbb{R}^{n} \rightarrow \mathbb{R}^{n}$ be cooperative and homogeneous and suppose the associated system

$$
\dot{x}(t)=f(x(t))
$$

is globally asymptotically stable. Then there exists some irreducible cooperative, homogeneous vector field $f_{1}: \mathbb{R}^{n} \rightarrow \mathbb{R}^{n}$ such that:

(i) $f_{1}(x) \geq f(x)$ for all $x \geq 0$;

(ii) $\dot{x}(t)=f_{1}(x(t))$ is globally asymptotically stable.

Proof: Choose any homogeneous, irreducible, order-preserving vector field $g: \mathbb{R}^{n} \rightarrow \mathbb{R}^{n}$. Note that for all $\epsilon>0, f+\epsilon g$ is cooperative, homogeneous and irreducible. We claim that for $\epsilon>0$ sufficiently small, the system $\dot{x}(t)=(f+\epsilon g)(x(t))$ will be globally asymptotically stable (GAS). Suppose this is not true. Then we can choose a sequence of positive real numbers $\epsilon_{n}$ such that $\epsilon_{n} \rightarrow 0$ as $n \rightarrow \infty$ for which $f+\epsilon_{n} g$ is not GAS for any $n$. It follows from Theorem 2.5 that there exist vectors $v_{n} \gg 0$ with $\left\|v_{n}\right\|=1$, and real numbers $\gamma_{n} \geq 0$ such that for all $n$

$$
\left(f+\epsilon_{n} g\right) v_{n}=\gamma_{n} v_{n}
$$

As $f$ and $g$ are continuous and $\left\|v_{n}\right\|=1$ for all $n$, it follows that there is some positive constant $M$ such that $\left\|\left(f+\epsilon_{n} g\right) v_{n}\right\| \leq M$ for all $n$. Hence the sequence $\gamma_{n}$ is bounded. As the sequences $v_{n}$ and $\gamma_{n}$ are bounded, by passing to subsequences if necessary, we can assume that $v_{n} \rightarrow v$ for some $v \geq 0,\|v\|=1$ and that $\gamma_{n} \rightarrow \gamma$ for some $\gamma \geq 0$. This together with the continuity of $f$ and $g$ implies that $f(v)=\gamma v$. This implies that the solution $x(\cdot, v)$ of the system $\dot{x}(t)=f(x(t))$ with $x(0)=v$ cannot tend to the equilibrium at 0 as $t \rightarrow \infty$, which contradicts our assumption that this original system is GAS. This shows that there must be some $\epsilon_{1}>0$ for which $\dot{x}(t)=\left(f+\epsilon_{1} g\right)(x(t))$ is GAS. It follows immediately that $f_{1}=f+\epsilon_{1} g$ satisfies (i) and (ii). 
From Theorem 2.1 we know that a positive LTI system $\dot{x}(t)=A x(t)$ is globally asymptotically stable if and only if there is some $v \gg 0$ such that $A v \ll 0$. Theorem 2.5 shows that for irreducible homogeneous cooperative systems, global asymptotic stability is equivalent to the existence of an eigenvector with negative eigenvalue. In the next result we show that the result of Theorem 2.1 extends to homogeneous cooperative systems without requiring irreducibility. Theorem 3.1: Let $f: \mathbb{R}^{n} \rightarrow \mathbb{R}^{n}$ be cooperative and homogeneous. Then the system

$$
\dot{x}(t)=f(x(t))
$$

is globally asymptotically stable if and only if there is some $v \gg 0$ such that $f(v) \ll 0$.

Proof: Suppose that $v \gg 0$ satisfies $f(v) \ll 0$. We shall first show that the trajectory $x(t, v)$ starting from the initial state $v$ tends to the equilibrium at zero as $t$ tends to infinity. As $f(v) \ll 0$ it follows that

$$
\left.\left(\frac{d x}{d t}(t, v)\right)\right|_{t=0} \ll 0
$$

and hence there is some $\delta>0$ such that

$$
x(t) \ll x(0)=v \text { for all } t \in(0, \delta] .
$$

In particular, $x(\delta, v) \ll v$ and thus there exists a real number $\alpha$ with $0<\alpha<1$ such that $x(\delta, v) \leq \alpha v$.

Now as as $f$ is cooperative it follows from Theorem 2.3 that

$$
\begin{aligned}
x(2 \delta, v) & =x(\delta, x(\delta, v)) \\
& \leq x(\delta, \alpha v) .
\end{aligned}
$$

Further, as $f$ is homogeneous this in turn implies that

$$
\begin{aligned}
x(2 \delta, v) & \leq x(\delta, \alpha v) \\
& =\alpha x(\delta, v) \leq \alpha^{2} v .
\end{aligned}
$$

Moreover, we can also conclude from the cooperativity of $f$ and (11) that $x(t) \ll \alpha v$ for all $t \in(\delta, 2 \delta)$. Iterating, we see that for $p=2,3, \ldots$

$$
\begin{aligned}
x(p \delta) & \leq \alpha^{p} v \\
x(t) & \ll \alpha^{p-1} v \text { for } t \in((p-1) \delta, p \delta) .
\end{aligned}
$$


and hence $x(t, v) \rightarrow 0$ as $t \rightarrow \infty$ as claimed.

Now, let $x_{0} \in \mathbb{R}_{+}^{n}$ be given. As $v \gg 0$, we can always choose some positive real number $K$ such that $x_{0} \ll K v$. As $f$ is assumed to be cooperative in $\mathbb{R}_{+}^{n}$, it follows from Theorem 2.3 that $x\left(t, x_{0}\right) \leq x(t, K v)=K x(t, v)$ for all $t \geq 0$. But it now follows from the above argument that $x\left(t, x_{0}\right) \rightarrow 0$ as $t \rightarrow \infty$. Hence, the system (10) is globally asymptotically stable as claimed. Conversely, suppose that (10) is globally asymptotically stable. By Lemma 3.1, we can choose some irreducible, homogeneous, cooperative mapping $f_{1}: \mathbb{R}^{n} \rightarrow \mathbb{R}^{n}$, satisfying

$$
f_{1}(x) \geq f(x) \text { for all } x \in \mathbb{R}_{+}^{n}
$$

such that the system $\dot{x}(t)=f_{1}(x(t))$ is GAS. Theorem 2.5 then implies that there is some vector $v \ll 0$ such that $f_{1}(v) \ll 0$. But $f(v) \leq f_{1}(v)$ and hence $f(v) \ll 0$ as well. This completes the proof.

Comment: In [4], a result similar to Theorem 3.1 for discrete-time systems has been presented. The authors of this manuscript have shown, under the assumption that $f: \mathbb{R}^{n} \rightarrow \mathbb{R}^{n}$ is irreducible and order-preserving, that $x(k+1)=f(x(k))$ is GAS if and only if there is no non-zero $w \in \mathbb{R}_{+}^{n}$ satisfying $f(w) \geq w$. Note that Theorem 3.1 combined with Proposition 3.1 establishes that in the continuous-time case, GAS is equivalent to there being no non-zero $w \in \mathbb{R}_{+}^{n}$ satisfying $f(w) \geq 0$.

Example 3.1: Consider the system $\dot{x}(t)=f(x(t))$ defined on $\mathbb{R}^{2}$ where

$$
f\left(x_{1}, x_{2}\right):=\left(\begin{array}{c}
2 x_{2}-x_{1}-\sqrt{x_{1}^{2}+x_{2}^{2}} \\
2 x_{1}-2 x_{2}-\sqrt{x_{1}^{2}+x_{2}^{2}}
\end{array}\right) .
$$

A phase portrait of this system is given in Figure 2 below. Two trajectories of the system are also illustrated, starting from the respective initial conditions $x^{(1)}:=\left(x_{1}^{(1)}, x_{2}^{(1)}\right)=(0.2,0.8)$ (solid line) and $x^{(2)}:=\left(x_{1}^{(2)}, x_{2}^{(2)}\right)=(0.8,0.2)$ (dashed line). Note that $f\left(x^{(i)}\right) \nless 0$ for $i=1,2$. However, it is clear from the figure that there exist $\left(x_{1}, x_{2}\right)$ such that $f\left(x_{1}, x_{2}\right) \ll 0$ (take for instance $x_{1}=x_{2}=\frac{1}{2}$ ). Hence by Theorem 3.1, the system $\dot{x}=f(x)$ is GAS.

Comment: For positive LTI systems it is well known that if $\dot{x}(t)=A x(t)$ is globally asymptotically stable, then $\dot{x}(t)=B x(t)$ will also be GAS for all Metzler matrices $B$ with $B \leq A$. The above result allows us to immediately draw the corresponding, known, conclusion for homogeneous cooperative systems. 


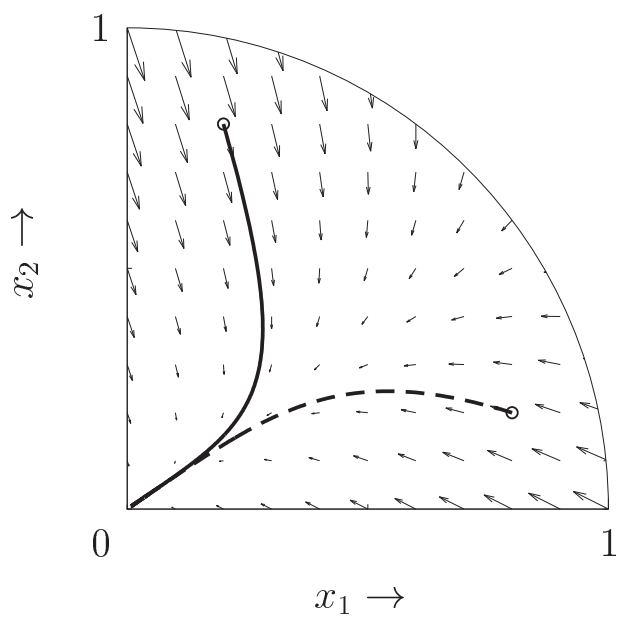

Fig. 1. Phase portrait of $f\left(x_{1}, x_{2}\right):=\left(2 x_{2}-x_{1}-\sqrt{x_{1}^{2}+x_{2}^{2}}, 2 x_{1}-2 x_{2}-\sqrt{x_{1}^{2}+x_{2}^{2}}\right)^{T}$.

Corollary 3.1: Let $f, g: \mathbb{R}^{n} \rightarrow \mathbb{R}^{n}$ be cooperative, homogeneous vector fields such that $g(x) \leq$ $f(x)$ for all $x \in \mathbb{R}_{+}^{n}$. If

$$
\dot{x}(t)=f(x(t))
$$

is globally asymptotically stable, then the system

$$
\dot{x}(t)=g(x(t))
$$

is also globally asymptotically stable.

Proof: As $\dot{x}(t)=f(x(t))$ is GAS, it follows from Theorem 3.1 that there is some $v \gg 0$ with $f(v) \ll 0$. But then, $g(v) \leq f(v) \ll 0$ and hence from Theorem 3.1, $\dot{x}(t)=g(x(t))$ is GAS also.

For a positive LTI system $\dot{x}(t)=A x(t)$, it is well known that global asymptotic stability implies that the diagonal entries of $A$ are all negative. The following simple corollary can be seen as an extension of this fact to homogeneous systems.

Corollary 3.2: Let $f: \mathbb{R}^{n} \mapsto \mathbb{R}^{n}$ be cooperative and homogeneous and suppose the system $\dot{x}=f(x)$ is globally asymptotically stable. Then there exists $w$ such that $\left.\frac{\partial f_{i}(\xi)}{\partial \xi_{i}}\right|_{\xi=w}<0$ for all $i$. 
Proof:Suppose the system is GAS; that is, suppose there exists $v \gg 0$ such that $f(v) \ll 0$. Then by Euler's Homogeneous Function Theorem we have that

$$
\left(\left.\sum_{j \neq i} \frac{\partial f_{i}(\xi)}{\partial \xi_{j}}\right|_{\xi=v} v_{j}\right)+\left.\frac{\partial f_{i}(\xi)}{\partial \xi_{i}}\right|_{\xi=v} v_{i}=f_{i}(v)
$$

Note that the first term on the left hand side of (12) is nonnegative by assumption of cooperativity. Now suppose $\left.\frac{\partial f_{i}(\xi)}{\partial \xi_{i}}\right|_{\xi=w} \geq 0$ for all $w$. Then it follows that $f_{i}(v) \geq 0$, which is a contradiction. This completes the proof.

Comment: Theorem 3.1 provides a test for the global asymptotic stability of a homogeneous cooperative system; If we can demonstrate the existence of a vector $v \gg 0$ with $f(v) \ll 0$, then the associated system is GAS. Conversely if no such vector exists, then the system is not GAS. However, it is typically much harder to show conclusively that no vector $v \gg 0$ satisfying $f(v) \ll 0$ exists. The following result, which can be thought of as a nonlinear theorem of the alternative for our situation, provides a way of demonstrating that a system is definitely not GAS.

Proposition 3.1: Let $f: \mathbb{R}^{n} \rightarrow \mathbb{R}^{n}$ be homogeneous and cooperative. Then exactly one of the following statements is true.

(i) There is some $v \gg 0$ with $f(v) \ll 0$;

(ii) There is some non-zero $w \geq 0$ with $f(w) \geq 0$.

Proof: We first show that at most one of these statements can be true. By way of contradiction, assume that there is some $v \gg 0$ such that $f(v) \ll 0$ and some non-zero $w \geq 0$ with $f(w) \geq 0$. Now let $\lambda=\max _{1 \leq i \leq n}\left(w_{i} / v_{i}\right)$. As $w \neq 0, \lambda>0$. Moreover, if we define $v^{\prime}=\lambda v$, it follows that $f\left(v^{\prime}\right) \ll 0, v^{\prime} \geq w$ and that there is some index $p$ for which $w_{p}=v_{p}^{\prime}$. Then for this $p$,

$$
\begin{aligned}
f_{p}\left(v^{\prime}\right)-f_{p}(w) & =\int_{0}^{1} \frac{d}{d s}\left(f_{p}\left(s v^{\prime}+(1-s) w\right)\right) d s \\
& =\int_{0}^{1}\left(\sum_{j=1}^{n} \frac{\partial f_{p}}{\partial x_{j}}\left(s v^{\prime}+(1-s) w\right)\left(v_{j}^{\prime}-w_{j}\right)\right) d s \\
& \geq 0,
\end{aligned}
$$

where the final inequality follows from the cooperativity of $f, v^{\prime} \geq w$ and $w_{p}=v_{p}^{\prime}$. But the above implies that

$$
0>f_{p}\left(v^{\prime}\right) \geq f_{p}(w) \geq 0
$$

which is a contradiction. 
Theorem 3.1 established that condition (i) is equivalent to $\dot{x}(t)=f(x(t))$ being GAS. Suppose that $\dot{x}(t)=f(x(t))$ is not GAS. The proof will be complete if we can show that under this assumption, there is some non-zero $w \geq 0$ with $f(w) \geq 0$. Let $g: \mathbb{R}^{n} \rightarrow \mathbb{R}^{n}$ be an irreducible, homogeneous, order-preserving vector field. Then it follows immediately from Corollary 3.1 that for any $\epsilon>0$, the system $\dot{x}(t)=(f+\epsilon g)(x(t))$ is not GAS. Now applying Theorem 2.5 and suitably adapting the argument of Lemma 3.1, we can conclude that there must exist some non-zero $w \geq 0$ with $f(w) \geq 0$.

Comment: The previous result provides a means of demonstrating that a homogeneous cooperative system is not GAS by showing the existence of a non-zero $w \geq 0$ with $f(w) \geq 0$.

The so-called D-stability property of positive LTI systems asserts that the global asymptotic stability of such systems is preserved under positive diagonal scaling. Theorem 3.1 allows us to immediately show that this property extends to homogeneous cooperative systems.

Theorem 3.2: Let $f: \mathbb{R}^{n} \rightarrow \mathbb{R}^{n}$ be cooperative and homogeneous. The system

$$
\dot{x}(t)=f(x(t))
$$

is globally asymptotically stable if and only if

$$
\dot{x}(t)=D f(x(t))
$$

is globally asymptotically stable for every diagonal matrix $D=\operatorname{diag}\left(d_{1}, \ldots, d_{n}\right)$ with $d_{i}>0$ for $1 \leq i \leq n$.

Proof: Suppose that (13) is globally asymptotically stable and let the diagonal matrix $D=$ $\operatorname{diag}\left(d_{1}, \ldots, d_{n}\right)$ with $d_{i}>0$ for $1 \leq i \leq n$ be given. Then by Theorem 3.1 it follows that there is some $v \gg 0$ such that $f(v) \ll 0$. But it is immediate that $D f(v) \ll 0$ and as $D f: \mathbb{R}^{n} \rightarrow \mathbb{R}^{n}$ is also homogeneous and cooperative it follows from Theorem 3.1 that the system (14) is globally asymptotically stable also. The converse direction is immediate.

\section{Stability of DELAYED COOPERATIVE SyStems}

In this section, we shall focus on extending the property of positive LTI systems described in Theorem 2.2 to cooperative homogeneous systems. As in the last section, unless explicitly stated otherwise, all vector fields are assumed to be continuous on $\mathbb{R}^{n}$ and $C^{1}$ on $\mathbb{R}^{n} \backslash\{0\}$.

The next result, which is the main result of this section is a direct extension of Theorem 2.2 to homogeneous systems. As it also immediately applies to positive linear systems, it provides 
an alternative view on the result of [8], which was established using a Lyapunov-Krasovskii functional. Our argument is based on the fundamental monotonicity property of the trajectories of the system.

Theorem 4.1: Let $f: \mathbb{R}^{n} \rightarrow \mathbb{R}^{n}$ and $g: \mathbb{R}^{n} \rightarrow \mathbb{R}^{n}$ be homogeneous and assume that $f$ is cooperative and $g$ is order-preserving. Then the time-delay system

$$
\dot{x}(t)=f(x(t))+g(x(t-\tau))
$$

is globally asymptotically stable for any $\tau \geq 0$ if and only if the undelayed system

$$
\dot{x}(t)=f(x(t))+g(x(t))
$$

is globally asymptotically stable.

Proof: If the system (15) is globally asymptotically stable (GAS) for any $\tau \geq 0$ then obviously it is GAS for $\tau=0$.

Conversely, suppose that (16) is GAS. Let $\tau \geq 0$ be given and for any $\phi \in C\left([-\tau, 0], \mathbb{R}_{+}^{n}\right)$, let $x(\cdot, \phi)$ denote the solution of (15) corresponding to the initial condition $\phi$. It follows from Theorem 3.1 that there is some vector $v \gg 0$ such that $(f+g)(v) \ll 0$. Let $\hat{v}$ denote the element of $C\left([-\tau, 0], \mathbb{R}_{+}^{n}\right)$ given by

$$
\hat{v}(s)=v \text { for }-\tau \leq s \leq 0 .
$$

The first step in the proof is to show that the solution $x(t, \hat{v})$ of (15) corresponding to the initial condition $\hat{v}$ converges asymptotically to the equilibrium point 0 as $t \rightarrow \infty$.

Now as

$$
\left.\frac{d}{d t}(x(t, \hat{v}))\right|_{t=0} \ll 0,
$$

as in the proof of Theorem 3.1, it follows that there is some $\delta>0$ such that $x(t, \hat{v}) \ll v$ for all $t \in(0, \delta]$. Further, if we write $x_{\delta, \hat{v}}$ for the element in $C\left([-\tau, 0], \mathbb{R}_{+}^{n}\right)$ given by

$$
x_{\delta, \hat{v}}(s)=x(\delta-s, \hat{v}) \text { for }-\tau \leq s \leq 0 \text {, }
$$

we can conclude that $x_{\delta, \hat{v}} \leq \hat{v}$.

Now as $f$ is cooperative and $g$ is order-preserving, it follows from Theorem 2.4 and the above observations that for all $s \in(0, \delta]$

$$
x(s+\delta, \hat{v})=x\left(s, x_{\delta, \hat{v}}\right) \leq x(s, \hat{v}) \ll \hat{v} .
$$


Repeating this process, we can conclude that for $k=2,3, \ldots$ and for all $s \in(0, \delta]$,

$$
x(s+k \delta, \hat{v}) \ll \hat{v}
$$

If we choose $k$ large enough to ensure that $t_{1}=k \delta>\tau$, then it follows that $x(s, \hat{v}) \ll v$ for $s \in\left[t_{1}-\tau, t_{1}\right]$. Hence, as $\left[t_{1}-\tau, t_{1}\right]$ is compact, there is some $\alpha$ with $0<\alpha<1$ such that

$$
x(s, \hat{v}) \leq \alpha v
$$

for all $s \in\left[t_{1}-\tau, t_{1}\right]$. In other words, $x_{t_{1}, \hat{v}} \leq \alpha \hat{v}$. Moreover, $x(s, \hat{v}) \leq \hat{v}$ for all $s \in\left[0, t_{1}\right]$. It now follows from Theorem 2.4 and the homogeneity of $f$ and $g$ that for $s \in\left[0, t_{1}\right]$,

$$
x\left(s+t_{1}, \hat{v}\right)=x\left(s, x_{t_{1}, \hat{v}}\right) \leq x(s, \alpha \hat{v})=\alpha x(s, \hat{v}) .
$$

Hence, $x_{2 t_{1}, \hat{v}} \leq \alpha x_{t_{1}, \hat{v}} \leq \alpha^{2} \hat{v}$. Iterating, we get that for $p=2,3,4, \ldots$

$$
x_{p t_{1}, \hat{v}} \leq \alpha^{p} \hat{v}
$$

and hence as $0<\alpha<1$, it follows that $x(t, \hat{v}) \rightarrow 0$ as $t \rightarrow \infty$.

To complete the proof, suppose we are given an initial condition $\phi \in C^{+}\left([-\tau, 0], \mathbb{R}_{+}^{n}\right)$. As $\phi$ is continuous and hence bounded on $[-\tau, 0]$, by choosing $M$ sufficiently large, we can ensure that $\phi \leq M \hat{v}$. As $f$ is cooperative and $g$ is order-preserving, it follows from Theorem 2.4 that $x(t, \phi) \leq x(t, M \hat{v})$. Moreover as $f$ and $g$ are homogeneous, $x(t, M \hat{v})=M x(t, \hat{v})$. It now follows immediately from the argument in the previous paragraph that $x(t, \phi) \rightarrow 0$ as $t \rightarrow \infty$.

Note that the argument given above also establishes that the system (15) is GAS if and only if there is some vector $v \gg 0$ such that $(f+g)(v) \ll 0$. In analogy with the undelayed case, this allows us to conclude the following.

Corollary 4.1: Let $f, f_{1}: \mathbb{R}^{n} \rightarrow \mathbb{R}^{n}$ and $g, g_{1}: \mathbb{R}^{n} \rightarrow \mathbb{R}^{n}$ be homogeneous vector fields. Assume that $f$ and $f_{1}$ are cooperative and that $g, g_{1}$ are order-preserving. Further assume that $\left(f_{1}+g_{1}\right)(x) \leq(f+g)(x)$ for all $x \in \mathbb{R}_{+}^{n}$. Then if the system

$$
\dot{x}(t)=f(x(t))+g(x(t-\tau))
$$

is globally asymptotically stable, then the system

$$
\dot{x(t)}=f_{1}(x(t))+g_{1}(x(t-\tau))
$$




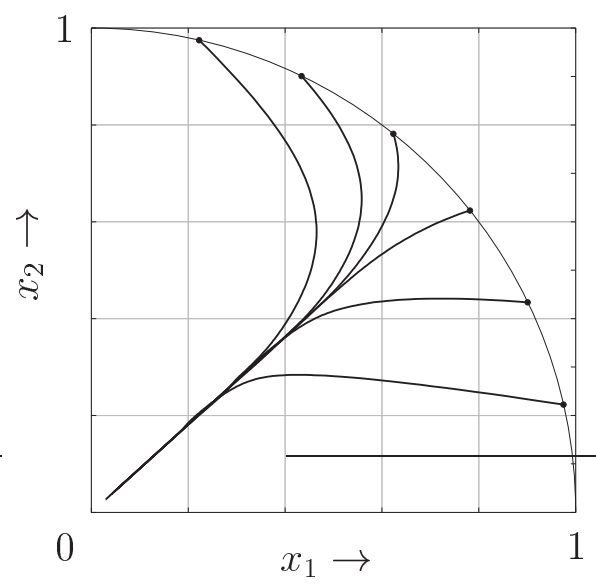

(a) $\tau=1$

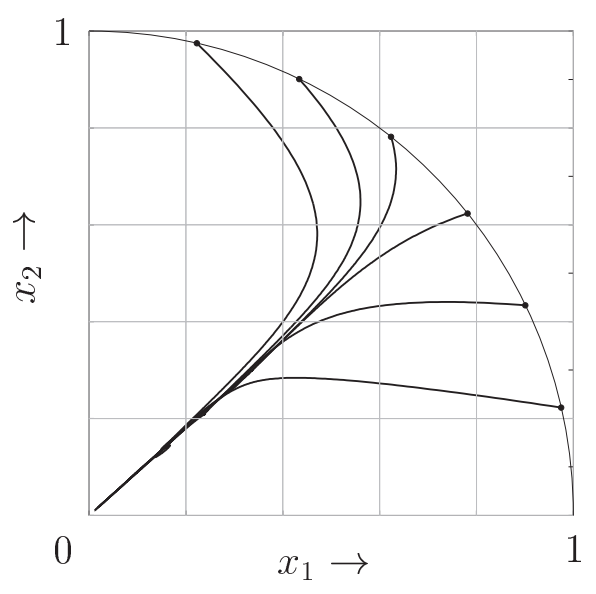

(b) $\tau=2$

Fig. 2. Trajectories of the system (21) for different state histories and different values of the delay parameter $\tau$.

is globally asymptotically stable also.

Example 4.1: Consider the delayed system:

$$
\dot{x}(t)=f(x(t))+g(x(t-\tau)),
$$

where $\tau \geq 0$ is a delay parameter,

$$
f\left(x_{1}, x_{2}\right):=\left(\begin{array}{cc}
-3 & 6 \\
2 & -2
\end{array}\right)\left(\begin{array}{l}
x_{1} \\
x^{2}
\end{array}\right)-\sqrt{x_{1}^{2}+x_{2}^{2}}\left(\begin{array}{l}
3 \\
1
\end{array}\right),
$$

and

$$
g\left(x_{1}, x_{2}\right):=\left(\begin{array}{c}
\frac{x_{1} x_{2}}{\sqrt{x_{1}^{2}+x_{2}^{2}}} \\
\frac{x_{1} x_{2}}{\sqrt{2 x_{1}^{2}+3 x_{2}^{2}}}
\end{array}\right) .
$$

It is straightforward to verify that this system satisfies the conditions of Theorem 4.1. Moreover, $(f+g)(1,1) \ll 0$. Hence, we can conclude the the origin is a GAS equilibrium of this system for any $\tau \geq 0$.

Several sample trajectories of the system (21) are shown in Figure 2. 
For $i=1, \ldots, 6$, let the initial conditions $x^{(\mathrm{a}, \mathrm{i})}$ and $x^{(\mathrm{b}, \mathrm{i})}$ be defined as follows:

$$
\begin{aligned}
& x^{(\mathrm{a}, \mathrm{i})}(s):=\left(\begin{array}{c}
1.1 \cos \left(\frac{\pi}{14} i\right)+0.1 \cos \left(2 \pi\left(t+\frac{3}{2}\right)-\frac{\pi}{14} i\right) \\
1.1 \sin \left(\frac{\pi}{14} i\right)-0.1 \sin \left(2 \pi\left(t+\frac{3}{2}\right)-\frac{\pi}{14} i\right)
\end{array}\right), \quad s \in[-1,0] ; \\
& x^{(\mathrm{b}, \mathrm{i})}(s):=\left(\begin{array}{c}
\cos \left(\frac{\pi}{14} i\right)+0.1 s(s+2) \\
\sin \left(\frac{\pi}{14} i\right)-0.1 s(s+2)^{2}
\end{array}\right), \quad s \in[-2,0] .
\end{aligned}
$$

The panel on the left shows 6 trajectories of the system (21) with $\tau=1$, corresponding to the initial conditions given by (24). The panel on the right shows 6 trajectories of the system (21) with $\tau=2$, corresponding to the initial conditions given by (25). It can be seen from the the figure that all trajectories converge to the origin as expected.

\section{Conclusions}

In this paper we have presented a number of results that extend fundamental properties of positive linear time-invariant (LTI) systems to a significant class of nonlinear positive systems. Specifically, we have shown that for homogeneous, cooperative systems, the D-stability property of positive LTI systems also holds. In addition, we have demonstrated that a homogeneous cooperative system subject to delay is globally asymptotically stable (GAS) for any non-negative delay if and only if it GAS for zero delay. This extends a result concerning the stability properties of delayed positive LTI systems recently published in [8]. While the result in this previous paper was derived using Lyapunov-Krasovskii arguments, our approach reveals the key role played by the monotonicity properties of the system trajectories. Examples have been presented to illustrate the main results. Future work will focus on extending the results here to broader classes of nonlinear positive systems.

\section{REFERENCES}

[1] D. Angeli and E. D. Sontag. Monotone control systems. IEEE Transactions on Automatic Control, 48(10):1684-1698, 2003.

[2] D. Angeli and E. D. Sontag. Multi-stability in monotone input/output systems. Systems and Control Letters, 51(3-4):185202,2004

[3] A. Berman and R.J. Plemmon. Non-negative matrices in the mathematical sciences. SIAM classics in applied mathematics, 1994.

[4] S. Dashkovskiy, B. Rüffer, and F. Wirth. An iss small-gain theorem for general networks. Technical Report 2005-05, Zentrum für Technomathematik, Universiteit Bremen, 2005. 
[5] P. de Leenheer and D. Aeyels. Extension of the perron-frobenius theorem to homogeneous systems. SIAM Journal of Control and Optimization, 41(2):563-582, 2002.

[6] L. Farina and S. Rinaldi. Positive Linear Systems. Wiley Interscience Series, 2000.

[7] S. Gaubert and J. Gunawardena. The Perron-Frobenius theorem for homogeneous monotone functions. Transactions of the American Mathematical Society, 356(12):4931-4950, 2004.

[8] W. M. Haddad and V. Chellaboina. Stability theory for nonnegative and compartmental dynamical systems with time delay. Systems and Control Letters, 51:355-361, 2004.

[9] W. M. Haddad and V. Chellaboina. Stability and dissipativity theory for nonnegative dynamical systems: a unified analysis framework for biological and physiological systems. Nonlinear Analysis: Real World Applications, 6:35-65, 2005.

[10] W. M. Haddad, T. Hayakawa, and J. M. Bailey. Adaptive control for nonlinear compartmental dynamical systems with applications to clinical pharmacology. Systems and Control Letters, 55:62-70, 2006.

[11] J. K Hale and S. M. Verduyn Lunel. Introduction to Functional Differential Equations. Springer-Verlag, 1993.

[12] M.W. Hirsch. Systems of differential equations that are competitive or cooperative. ii: Convergence almost everywhere. SIAM Journal of Mathematical Analysis, 16:423-439, 1985.

[13] M. Morishima. Equilibrium, Stability and Growth - A Multi-sectorial Analysis. Oxford University Press, 1964.

[14] P. H. A. Ngoc. A Perron-Frobenius theorem for a class of positive quasi-polynomial matrices. Applied Mathematics Letters, 19(8):747-751, 2006.

[15] R. D. Nussbaum. Hilbert's Projective Metric and Iterated Nonlinear Maps. Memoirs of the American Mathematical Society, 1988.

[16] H. Smith. Monotone Dynamical Systems, an Introduction to the Theory of Competitive and Cooperative Systems. AMS Mathematical Surveys and Monographs, 1995. 